Supporting information

\title{
Structures of Metal-Organic Frameworks with Rod Secondary Building Units
}

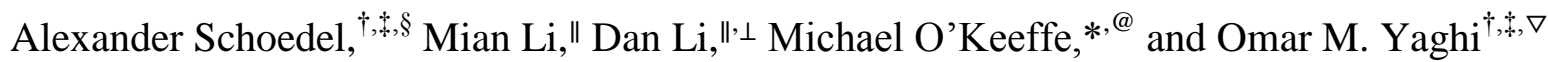

${ }^{\dagger}$ Department of Chemistry, University of California, Berkeley, California 94720, United States

${ }^{\ddagger}$ Materials Sciences Division, Lawrence Berkeley National Laboratory, Kavli Energy

Nanoscience Institute, Berkeley, California 94720, United States

${ }^{\S}$ Department of Chemistry, Florida Institute of Technology, 150 West University Boulevard, Melbourne, Florida 32901, United States

"Department of Chemistry, Shantou University, Guangdong 515063, P. R. China

${ }^{\perp}$ College of Chemistry and Materials Science, Jinan University, Guangzhou 510632, P. R. China

${ }^{\circledR}$ School of Molecular Sciences, Arizona State University, Tempe, Arizona 85287, United States

${ }^{\nabla}$ King Abdulaziz City for Science and Technology, P.O Box 6086, Riyadh 11442, Saudi Arabia 
Systre output files for nets with maximum symmetry embedding (no three letter code in RCSR assigned)

\section{Rod net-1 (rn-1):}

\section{CRYSTAL}

NAME "ICC2010 Eu-\#1"

GROUP Pnma

CELL 1.67379 6.101713.4759990.000090.000090.0000

NODE $17 \quad 0.247440 .004500 .33181$

NODE 260.247430 .079690 .08016

NODE 360.285740 .167660 .32497

EDGE $0.247440 .004500 .33181 \quad 0.747440 .004500 .16819$

EDGE $0.247430 .079690 .08016-0.214260 .167660 .17503$

EDGE $0.247430 .079690 .08016 \quad 0.25256-0.00450-0.16819$

EDGE $0.247440 .004500 .33181 \quad 0.285740 .167660 .32497$

EDGE $0.285740 .167660 .32497 \quad 0.285740 .332340 .32497$

EDGE $0.247430 .079690 .08016-0.252560 .004500 .16819$

EDGE $0.285740 .167660 .32497 \quad 0.785740 .167660 .17503$

EDGE $0.247430 .079690 .08016 \quad 0.247440 .004500 .33181$

EDGE $0.247430 .079690 .08016 \quad 0.747440 .004500 .16819$

EDGE $0.247430 .079690 .08016 \quad 0.285740 .167660 .32497$

\# EDGE_CENTER 0.497440 .004500 .25000

\# EDGE_CENTER 0.016580 .123670 .12760

\# EDGE_CENTER $0.249990 .03759-0.04402$

\# EDGE_CENTER 0.266590 .086080 .32839

\# EDGE_CENTER 0.28574 0.25000 0.32497

\# EDGE_CENTER -0.00257 0.04210 0.12418

\# EDGE_CENTER 0.535740 .167660 .25000

\# EDGE_CENTER 0.247430 .042100 .20598

\# EDGE_CENTER 0.497430 .042100 .12418

\# EDGE_CENTER 0.266580 .123670 .20256

END 


\section{Rod net-2 (rn-2):}

CRYSTAL

NAME "IC2009 Pr-1-\#1"

GROUP A12/n1

CELL 3.99051 1.648354.3835490.000091.149690.0000

NODE 170.130570 .228550 .06470

NODE 260.379510 .233630 .06169

NODE 360.252200 .222990 .63453

EDGE $0.379510 .233630 .06169 \quad 0.247800 .22299-0.13453$

EDGE $0.130570 .228550 .06470 \quad 0.120490 .73363-0.06169$

EDGE 0.379510 .233630 .061690 .252200 .722990 .13453

EDGE $0.130570 .228550 .06470 \quad 0.247800 .22299-0.13453$

EDGE $0.130570 .228550 .06470 \quad 0.379510 .233630 .06169$

EDGE $0.130570 .228550 .06470 \quad 0.25220-0.277010 .13453$

EDGE $0.252200 .222990 .63453 \quad 0.247800 .222990 .86547$

EDGE $0.130570 .228550 .06470 \quad 0.12049-0.26637-0.06169$

EDGE $0.130570 .228550 .06470 \quad 0.252200 .722990 .13453$

EDGE $0.130570 .228550 .06470-0.120490 .266370 .06169$

\# EDGE_CENTER $0.313650 .22831-0.03642$

\# EDGE_CENTER 0.125530 .481090 .00151

\# EDGE_CENTER 0.315850 .478310 .09811

\# EDGE_CENTER 0.189190 .22577 -0.03491

\# EDGE_CENTER 0.25504 0.231090.06320

\# EDGE_CENTER 0.19139 -0.024230.09961

\# EDGE_CENTER 0.25000 0.222990.75000

\# EDGE_CENTER 0.12553 -0.018910.00151

\# EDGE_CENTER 0.191390.475770.09961

\# EDGE_CENTER 0.005040 .247460 .06320

END 


\section{Rod net-3 (rn-3):}

\section{CRYSTAL}

NAME "CC2012 La-\#1"

GROUP I121

CELL 3.26685 1.63597 6.3644290.000092.501590.0000

NODE 170.093970 .173220 .20385

NODE 270.399760 .175740 .20526

NODE $37 \quad 0.256020 .174270 .34005$

NODE 440.252200 .123130 .49725

NODE 540.356310 .373260 .63261

NODE 640.589690 .051630 .06544

NODE 740.112600 .000000 .63064

EDGE $0.112600 .000000 .63064 \quad 0.252200 .123130 .49725$

EDGE $0.252200 .123130 .49725 \quad 0.089690 .551630 .56544$

EDGE $0.589690 .051630 .06544 \quad 0.85631-0.126740 .13261$

EDGE $0.252200 .123130 .49725 \quad 0.356310 .373260 .63261$

EDGE $0.093970 .173220 .20385 \quad 0.10024-0.324260 .29474$

EDGE 0.112600 .000000 .630640 .089690 .551630 .56544

EDGE $0.093970 .173220 .20385 \quad 0.100240 .675740 .29474$

EDGE $0.112600 .000000 .63064 \quad 0.356310 .373260 .63261$

EDGE $0.093970 .173220 .20385 \quad 0.24398-0.325730 .15995$

EDGE $0.093970 .173220 .20385 \quad 0.243980 .674270 .15995$

EDGE $0.589690 .051630 .06544 \quad 0.410310 .05163-0.06544$

EDGE $0.093970 .173220 .20385 \quad 0.256020 .174270 .34005$

EDGE $0.093970 .173220 .20385 \quad 0.399760 .175740 .20526$

EDGE $0.256020 .174270 .34005 \quad 0.399760 .175740 .20526$

EDGE $0.093970 .173220 .20385 \quad-0.14369-0.126740 .13261$

EDGE $0.256020 .174270 .34005 \quad 0.10024-0.324260 .29474$

EDGE $0.256020 .174270 .34005 \quad 0.100240 .675740 .29474$

EDGE $0.256020 .174270 .34005 \quad 0.252200 .123130 .49725$

EDGE $0.112600 .000000 .63064-0.10024-0.324260 .70526$ \# EDGE_CENTER 0.182400 .061560 .56395 
\# EDGE_CENTER 0.170950 .337380 .53135

\# EDGE_CENTER 0.72300 -0.03756 0.09902

\# EDGE_CENTER 0.30426 0.248190.56493

\# EDGE_CENTER $0.09710-0.075520 .24929$

\# EDGE_CENTER 0.101150 .275810 .59804

\# EDGE_CENTER 0.097100 .424480 .24929

\# EDGE_CENTER 0.234450 .186630 .63163

\# EDGE_CENTER $0.16898-0.076260 .18190$

\# EDGE_CENTER 0.168980 .423740 .18190

\# EDGE_CENTER 0.500000 .051630 .00000

\# EDGE_CENTER 0.174990 .173740 .27195

\# EDGE_CENTER 0.246860 .174480 .20456

\# EDGE_CENTER 0.327890 .175000 .27266

\# EDGE_CENTER -0.02486 0.023240.16823

\# EDGE_CENTER 0.17813 -0.07500 0.31739

\# EDGE_CENTER 0.178130 .425000 .31739

\# EDGE_CENTER 0.254110 .148700 .41865

\# EDGE_CENTER 0.00618 -0.162130.66795

END

\section{Rod net-4 (rn-4):}

CRYSTAL

NAME testa-

GROUP I12/a1

CELL 1.76113 6.57320 5.3245390.000091.606090.0000

NODE 130.509010 .244110 .32787

NODE 230.173460 .129720 .08957

NODE 330.066270 .128500 .27287

NODE 450.583380 .113940 .42316

NODE 580.074680 .037490 .42412

EDGE $0.074680 .037490 .42412-0.07468-0.037490 .57588$

EDGE 0.074680 .037490 .424120 .425320 .037490 .57588

EDGE $0.066270 .128500 .27287-0.009010 .255890 .17213$

EDGE $0.074680 .037490 .42412 \quad 0.57468-0.037490 .42412$ 
EDGE $0.509010 .244110 .32787 \quad 0.583380 .113940 .42316$

EDGE $0.074680 .037490 .424120 .08338-0.113940 .42316$

EDGE $0.074680 .037490 .42412-0.083380 .113940 .57684$

EDGE $0.074680 .037490 .42412-0.416620 .113940 .42316$

EDGE $0.583380 .113940 .42316 \quad 0.916620 .113940 .57684$

EDGE $0.066270 .128500 .27287 \quad 0.074680 .037490 .42412$

EDGE $0.173460 .129720 .08957-0.009010 .255890 .17213$

EDGE $0.173460 .129720 .08957 \quad 0.326540 .12972-0.08957$

EDGE $0.173460 .129720 .08957 \quad 0.066270 .128500 .27287$

\# EDGE_CENTER -0.00000 -0.00000 0.50000

\# EDGE_CENTER 0.250000 .037490 .50000

\# EDGE_CENTER 0.028630 .192200 .22250

\# EDGE_CENTER $0.32468-0.000000 .42412$

\# EDGE_CENTER 0.546200 .179020 .37551

\# EDGE_CENTER 0.07903 -0.03822 0.42364

\# EDGE_CENTER -0.004350.075720.50048

\# EDGE_CENTER -0.170970.075720.42364

\# EDGE_CENTER 0.750000 .113940 .50000

\# EDGE_CENTER 0.070480 .083000 .34849

\# EDGE_CENTER 0.082220 .192810 .13085

\# EDGE_CENTER 0.250000 .129720 .00000

\# EDGE_CENTER 0.119870 .129110 .18122

END

\section{Rod net-5 (rn-5):}

CRYSTAL

NAME "IC2007 Tb-\#1"

GROUP Pbcn

CELL 4.71559 1.81037 3.5611290.000090.000090.0000

NODE $15 \quad 0.295060 .248030 .22514$

NODE 250.310960 .099020 .49472

NODE 350.098900 .099020 .49472

EDGE $0.295060 .24803 \quad 0.22514 \quad 0.401100 .40098-0.00528$

EDGE $0.098900 .099020 .494720 .18904-0.400980 .49472$ 
EDGE 0.098900 .099020 .494720 .189040 .599020 .49472

EDGE $0.295060 .24803 \quad 0.22514 \quad 0.20494-0.251970 .22514$

EDGE $0.295060 .248030 .22514 \quad 0.189040 .40098-0.00528$

EDGE $0.295060 .248030 .22514 \quad 0.310960 .099020 .49472$

EDGE $0.098900 .099020 .49472-0.09890-0.099020 .50528$

EDGE 0.098900 .099020 .494720 .310960 .099020 .49472

\# EDGE_CENTER 0.348080 .324510 .10993

\# EDGE_CENTER 0.14397 -0.15098 0.49472

\# EDGE_CENTER 0.143970 .349020 .49472

\# EDGE_CENTER $0.25000-0.001970 .22514$

\# EDGE_CENTER 0.242050 .324510 .10993

\# EDGE_CENTER 0.303010 .173530 .35993

\# EDGE_CENTER -0.00000 -0.00000 0.50000

\# EDGE_CENTER 0.204930 .099020 .49472

END

\section{Rod net-6 (rn-6):}

CRYSTAL

NAME "QiuS1 IC2005-\#1"

GROUP C12/c1

CELL 3.55123 3.071193.2568490.0000 108.704890.0000

NODE 150.339210 .152540 .39285

NODE 260.148730 .402720 .30059

NODE 350.363480 .150860 .00583

EDGE $0.363480 .150860 .00583 \quad 0.136520 .34914-0.00583$

EDGE 0.148730 .402720 .300590 .160790 .652540 .10715

EDGE $0.363480 .150860 .00583 \quad 0.660790 .152540 .10715$

EDGE $0.148730 .402720 .30059 \quad 0.339210 .152540 .39285$

EDGE $0.363480 .150860 .00583 \quad 0.35127-0.097280 .19941$

EDGE $0.363480 .150860 .00583 \quad 0.33921-0.15254-0.10715$

EDGE $0.148730 .402720 .30059-0.148730 .402720 .19941$

EDGE $0.363480 .150860 .00583 \quad 0.351270 .09728-0.30059$

EDGE $0.148730 .402720 .30059 \quad 0.160790 .347460 .60715$

\# EDGE_CENTER $0.250000 .25000-0.00000$ 
\# EDGE_CENTER 0.154760 .527630 .20387

\# EDGE_CENTER 0.512130 .151700 .05649

\# EDGE_CENTER 0.243970 .277630 .34672

\# EDGE_CENTER 0.357370 .026790 .10262

\# EDGE_CENTER 0.35135 -0.00084 -0.05066

\# EDGE_CENTER -0.00000 0.402720.25000

\# EDGE_CENTER $0.357370 .12407-0.14738$

\# EDGE_CENTER 0.154760 .375090 .45387

END

\section{Rod net-7 (rn-7):}

CRYSTAL

NAME MOF-80-\#1

GROUP P-1

CELL 1.95228 1.97220 1.99525 97.177695.5206 101.1282

NODE 160.471930 .464660 .24259

NODE 260.615110 .057680 .79688

NODE 350.142770 .165950 .85779

EDGE $0.471930 .464660 .24259 \quad 0.142770 .16595-0.14221$

EDGE 0.471930 .464660 .242590 .857230 .834050 .14221

EDGE $0.471930 .464660 .24259 \quad 0.384890 .942320 .20312$

EDGE $0.471930 .464660 .24259 \quad 0.528070 .53534-0.24259$

EDGE $0.471930 .464660 .24259 \quad 0.38489-0.057680 .20312$

EDGE 0.142770 .165950 .857790 .615110 .057680 .79688

EDGE $0.142770 .165950 .85779-0.384890 .057680 .79688$

EDGE $0.471930 .464660 .24259 \quad 0.528070 .535340 .75741$

EDGE $0.615110 .057680 .79688 \quad 0.38489-0.057681 .20312$

EDGE $0.142770 .165950 .85779 \quad 0.38489-0.057681 .20312$

\# EDGE_CENTER 0.307350 .315310 .05019

\# EDGE_CENTER 0.664580 .649350 .19240

\# EDGE_CENTER 0.428410 .703490 .22286

\# EDGE_CENTER 0.500000 .500000 .00000

\# EDGE_CENTER 0.428410 .203490 .22286

\# EDGE_CENTER 0.378940 .111820 .82734 
\# EDGE_CENTER -0.12106 0.111820.82734

\# EDGE_CENTER 0.500000 .500000 .50000

\# EDGE_CENTER 0.500000 .000001 .00000

\# EDGE_CENTER 0.263830 .054141 .03046

\section{Rod net-8 (rn-8):}

CRYSTAL

NAME "CGD2010 La-\#1"

GROUP C12/c1

CELL 3.80095 6.281951.9973390.0000 103.670290.0000

NODE 130.135290 .090500 .30851

NODE 330.000000 .228290 .25000

NODE 460.133570 .507300 .09200

NODE 650.000000 .387660 .25000

EDGE $0.135290 .090500 .30851 \quad 0.366430 .007300 .40800$

EDGE $0.133570 .507300 .09200 \quad 0.133570 .49270-0.40800$

EDGE $0.133570 .507300 .09200 \quad-0.133570 .49270-0.09200$

EDGE $0.135290 .090500 .30851-0.135290 .090500 .19149$

EDGE $0.000000 .387660 .25000 \quad 0.133570 .492700 .59200$

EDGE $0.000000 .387660 .25000 \quad 0.133570 .507300 .09200$

EDGE $0.00000 \quad 0.228290 .25000 \quad 0.000000 .387660 .25000$

EDGE $0.00000 \quad 0.228290 .25000 \quad 0.135290 .090500 .30851$

\# EDGE_CENTER 0.250860 .048900 .35826

\# EDGE_CENTER $0.133570 .50000-0.15800$

\# EDGE_CENTER 0.000000 .500000 .00000

\# EDGE_CENTER 0.000000 .090500 .25000

\# EDGE_CENTER 0.066790 .440180 .42100

\# EDGE_CENTER 0.066790 .447480 .17100

\# EDGE_CENTER 0.000000 .307970 .25000

\# EDGE_CENTER 0.067640 .159400 .27925

END 


\author{
Rod net-9 (rn-9): \\ CRYSTAL \\ NAME "IC2005 ZnNa-\#1" \\ GROUP C12/c1 \\ CELL 2.67599 2.735662.0000090.0000 110.862290.0000 \\ NODE 180.199950 .000000 .34528 \\ NODE 260.500000 .241520 .00000 \\ EDGE $0.500000 .241520 .00000 \quad 0.500000 .241520 .50000$ \\ EDGE $0.199950 .000000 .34528 \quad 0.000000 .258480 .50000$ \\ EDGE $0.199950 .000000 .34528-0.000000 .25848-0.00000$ \\ EDGE $0.199950 .000000 .34528-0.00000-0.258480 .50000$ \\ EDGE $0.199950 .000000 .34528 \quad 0.00000-0.258480 .00000$ \\ EDGE $0.199950 .000000 .34528-0.199950 .000000 .15472$ \\ EDGE $0.199950 .000000 .34528 \quad 0.500000 .241520 .50000$ \\ EDGE $0.199950 .000000 .34528 \quad 0.19995-0.00000-0.15472$ \\ \# EDGE_CENTER 0.500000 .241520 .25000 \\ \# EDGE_CENTER 0.099980 .129240 .42264 \\ \# EDGE_CENTER 0.099980 .129240 .17264 \\ \# EDGE_CENTER $0.09998-0.129240 .42264$ \\ \# EDGE_CENTER $0.09998-0.129240 .17264$ \\ \# EDGE_CENTER 0.000000 .000000 .25000 \\ \# EDGE_CENTER 0.349980 .120760 .42264 \\ \# EDGE_CENTER 0.199950 .000000 .09528 \\ END
}

\title{
Rod net-b (rn-b):
}

CRYSTAL

NAME MOF-79-\#1

GROUP I12/m1

CELL 2.477513.33976 3.2670890.0000 111.724090.0000

NODE 150.344040 .149650 .06302

NODE 240.036050 .149640 .19189

EDGE $0.344040 .149650 .06302 \quad 0.655960 .14965-0.06302$ 
EDGE $0.036050 .149640 .19189-0.344040 .14965-0.06302$

EDGE $0.344040 .149650 .06302 \quad 0.34404-0.149650 .06302$

EDGE $0.036050 .149640 .191890 .03605-0.149640 .19189$

EDGE 0.036050 .149640 .191890 .155960 .350350 .43698

EDGE 0.036050 .149640 .191890 .344040 .149650 .06302

\# EDGE_CENTER 0.500000 .149650 .00000

\# EDGE_CENTER -0.15399 0.149640.06443

\# EDGE_CENTER 0.344040 .000000 .06302

\# EDGE_CENTER 0.036050 .000000 .19189

\# EDGE_CENTER 0.096010 .250000 .31443

\# EDGE_CENTER 0.190040 .149640 .12746

END

\section{Rod net-10 (rn-10):}

CRYSTAL

NAME CEC2011-\#1

GROUP I12/a1

CELL 4.38362 3.91022 14.9501390.000097.635790.0000

NODE 150.250000 .321990 .00000

NODE 240.030470 .333800 .01162

NODE 340.104700 .011820 .46588

NODE 430.146670 .203770 .27898

NODE 530.124840 .313500 .21807

NODE 630.070100 .016430 .39838

NODE 730.114020 .111420 .33934

NODE 830.032420 .416370 .15946

NODE 930.250000 .065750 .00000

NODE 1030.135030 .655880 .49616

EDGE $0.250000 .065750 .00000 \quad 0.250000 .321990 .00000$

EDGE $0.135030 .655880 .49616 \quad 0.364970 .655880 .50384$

EDGE $0.250000 .321990 .00000 \quad 0.104700 .48818-0.03412$

EDGE $0.114020 .111420 .33934 \quad 0.146670 .203770 .27898$

EDGE $0.250000 .321990 .00000 \quad 0.030470 .333800 .01162$

EDGE $0.146670 .203770 .27898 \quad 0.124840 .313500 .21807$ 
EDGE $0.114020 .111420 .33934-0.03242-0.083630 .34054$

EDGE $0.030470 .333800 .01162-0.135030 .155880 .00384$

EDGE $0.030470 .333800 .01162-0.104700 .511820 .03412$

EDGE $0.250000 .065750 .00000 \quad 0.13503-0.15588-0.00384$

EDGE $0.030470 .333800 .011620 .104700 .48818-0.03412$

EDGE $0.070100 .016430 .39838 \quad 0.104700 .011820 .46588$

EDGE $0.114020 .111420 .33934 \quad 0.070100 .016430 .39838$

EDGE $0.070100 .016430 .39838-0.03242-0.083630 .34054$

EDGE $0.146670 .203770 .27898 \quad 0.375160 .186500 .28193$

EDGE $0.124840 .313500 .21807 \quad 0.032420 .416370 .15946$

\# EDGE_CENTER 0.250000 .193870 .00000

\# EDGE_CENTER 0.250000 .655880 .50000

\# EDGE_CENTER 0.177350 .40509 -0.01706

\# EDGE_CENTER 0.130350 .157590 .30916

\# EDGE_CENTER 0.140230 .327900 .00581

\# EDGE_CENTER 0.135760 .258640 .24853

\# EDGE_CENTER 0.040800 .013890 .33994

\# EDGE_CENTER -0.05228 0.244840.00773

\# EDGE_CENTER -0.037120.422810.02287

\# EDGE_CENTER 0.19251-0.04506 -0.00192

\# EDGE_CENTER $0.067590 .41099-0.01125$

\# EDGE_CENTER 0.087400 .014120 .43213

\# EDGE_CENTER 0.092060 .063930 .36886

\# EDGE_CENTER 0.01884 -0.03360 0.36946

\# EDGE_CENTER 0.260910 .195130 .28046

\# EDGE_CENTER 0.078630 .364930 .18876

END

\section{Rod net-11 (rn-11):}

CRYSTAL

NAME rod54

GROUP P-3

CELL 20.10449 20.10449 1.9080490.000090.0000120.0000

NODE 11130.228340 .147360 .40685 
NODE $1123 \quad 0.127930 .228020 .42104$

NODE 11330.087340 .222030 .08007

NODE 12130.188070 .063580 .44348

NODE 12230.088380 .189940 .20462

NODE 12330.137820 .194040 .04498

NODE 13130.287620 .032890 .21595

NODE 13230.343020 .057580 .07552

NODE 13330.259570 .302290 .29192

NODE 14130.483670 .265820 .43376

NODE $1423 \quad 0.252860 .478880 .22062$

NODE $1433 \quad 0.203540 .474970 .05733$

NODE 15130.442550 .180260 .40238

NODE $1523 \quad 0.214940 .441970 .42121$

NODE 15330.253740 .443530 .08069

NODE $1613 \quad 0.377730 .416210 .21406$

NODE 16230.409410 .024450 .29575

NODE 16330.401770 .384860 .06831

NODE 17130.145380 .599500 .43319

NODE 17230.532560 .139000 .05517

NODE 17330.583570 .146860 .21370

NODE 18130.542670 .104430 .44259

NODE 18230.107540 .515930 .39552

NODE 18330.578740 .102270 .09207

NODE 19130.323450 .259590 .13811

NODE 19230.087900 .364360 .22988

NODE $1933 \quad 0.378890 .287280 .27781$

NODE 1050.178530 .229560 .41167

NODE 1150.266910 .238250 .07830

NODE 1250.037430 .187210 .25493

NODE 1370.227440 .245040 .24537

NODE 1490.230420 .009180 .25467

NODE 1570.044190 .233510 .08810

NODE 1690.225080 .195520 .41205

NODE 1790.186370 .191670 .07868 
NODE 1870.183250 .011500 .42128

NODE 1950.400530 .086220 .04667

NODE 2050.490390 .098360 .37978

NODE 2150.302100 .478250 .28660

NODE 2290.351080 .446750 .28699

NODE 2370.441130 .081240 .21307

NODE 2490.441500 .129830 .37977

NODE 2570.297180 .432660 .12040

NODE 2690.480830 .135100 .04629

NODE 2770.495320 .143940 .54646

NODE 2850.164020 .439230 .35854

NODE 2950.073660 .426240 .02435

NODE 3050.516460 .365420 .30848

NODE 3190.436310 .316050 .30817

NODE 3270.114640 .421770 .19167

NODE 3390.154090 .475750 .02523

NODE 3470.168590 .484670 .52507

NODE 3570.475630 .370000 .14136

NODE 3690.114530 .470030 .35833

EDGE $0.114640 .421770 .19167 \quad 0.154090 .475750 .02523$

EDGE $0.400530 .086220 .04667 \quad 0.446750 .09567-0.28699$

EDGE $0.114640 .421770 .19167 \quad 0.114530 .470030 .35833$

EDGE $0.583570 .146860 .21370 \quad 0.545880 .145380 .56681$

EDGE $0.186370 .191670 .07868 \quad 0.171750 .183250 .57872$

EDGE 0.532560 .139000 .055170 .545880 .145380 .56681

EDGE $0.495320 .143940 .54646 \quad 0.545880 .145380 .56681$

EDGE $0.436310 .316050 .30817 \quad 0.484670 .316080 .47493$

EDGE $0.186370 .191670 .07868 \quad 0.171750 .18325-0.42128$

EDGE $0.154090 .475750 .02523 \quad 0.120260 .43631-0.30817$

EDGE $0.532560 .139000 .05517 \quad 0.583570 .146860 .21370$

EDGE $0.400530 .086220 .04667 \quad 0.441500 .129830 .37977$

EDGE $0.114530 .470030 .35833 \quad 0.120260 .436310 .69183$

EDGE $0.475630 .370000 .14136 \quad 0.436310 .316050 .30817$

EDGE $0.400530 .086220 .04667 \quad 0.441130 .081240 .21307$ 
EDGE $0.583570 .146860 .21370 \quad 0.634580 .151040 .30848$

EDGE 0.480830 .135100 .046290 .532560 .139000 .05517

EDGE $0.114640 .421770 .19167 \quad 0.120260 .436310 .69183$

EDGE $0.044190 .233510 .08810 \quad 0.029560 .22508-0.41205$

EDGE $0.114640 .421770 .19167 \quad 0.120260 .43631-0.30817$

EDGE $0.409410 .024450 .295750 .38486-0.01691-0.06831$

EDGE $0.377730 .416210 .21406 \quad 0.351080 .446750 .28699$

EDGE $0.516460 .365420 .30848 \quad 0.484670 .316080 .47493$

EDGE $0.044190 .233510 .08810 \quad 0.029560 .225080 .58795$

EDGE $0.186370 .191670 .07868 \quad 0.225080 .195520 .41205$

EDGE $0.154090 .475750 .02523 \quad 0.151040 .51646-0.30848$

EDGE $0.441130 .081240 .21307 \quad 0.409410 .024450 .29575$

EDGE $0.183250 .011500 .42128 \quad 0.19552-0.029560 .58795$

EDGE $0.044190 .233510 .08810 \quad-0.005310 .18637-0.07868$

EDGE $0.114530 .470030 .35833 \quad 0.151040 .516460 .69152$

EDGE $0.073660 .426240 .02435 \quad 0.016910 .40177-0.06831$

EDGE $0.475630 .370000 .14136 \quad 0.516460 .365420 .30848$

EDGE $0.073660 .426240 .02435 \quad 0.114530 .470030 .35833$

EDGE $0.441500 .129830 .37977 \quad 0.442550 .180260 .40238$

EDGE $0.186370 .191670 .07868 \quad 0.221240 .23042-0.25467$

EDGE $0.253740 .443530 .08069 \quad 0.214940 .441970 .42121$

EDGE $0.073660 .426240 .02435 \quad 0.120260 .43631-0.30817$

EDGE $0.227440 .245040 .24537 \quad 0.225080 .195520 .41205$

EDGE $0.073660 .426240 .024350 .105630 .47563-0.14136$

EDGE $0.073660 .426240 .02435 \quad 0.114640 .421770 .19167$

EDGE $0.164020 .439230 .35854 \quad 0.214940 .441970 .42121$

EDGE $0.253740 .443530 .08069 \quad 0.297180 .432660 .12040$

EDGE $0.044190 .233510 .08810-0.009180 .221240 .25467$

EDGE $0.230420 .009180 .25467 \quad 0.183250 .011500 .42128$

EDGE $0.164020 .439230 .35854 \quad 0.168590 .484670 .52507$

EDGE $0.230420 .009180 .25467 \quad 0.19552-0.029560 .58795$

EDGE 0.401770 .384860 .068310 .377730 .416210 .21406

EDGE $0.409410 .024450 .29575 \quad 0.416210 .03848-0.21406$

EDGE $0.483670 .265820 .43376 \quad 0.484670 .316080 .47493$ 
EDGE $0.037430 .187210 .25493 \quad 0.029560 .225080 .58795$

EDGE $0.154090 .475750 .02523 \quad 0.164020 .439230 .35854$

EDGE $0.203540 .474970 .05733 \quad 0.252860 .478880 .22062$

EDGE $0.186370 .191670 .07868 \quad 0.187210 .14978-0.25493$

EDGE $0.114640 .421770 .19167 \quad 0.164020 .439230 .35854$

EDGE $0.164020 .439230 .35854 \quad 0.120260 .436310 .69183$

EDGE $0.252860 .478880 .22062 \quad 0.302100 .478250 .28660$

EDGE 0.480830 .135100 .046290 .495320 .143940 .54646

EDGE $0.154090 .475750 .02523 \quad 0.203540 .474970 .05733$

EDGE $0.186370 .191670 .07868 \quad 0.227440 .245040 .24537$

EDGE $0.442550 .180260 .40238 \quad 0.441970 .227030 .57879$

EDGE $0.044190 .233510 .08810 \quad 0.037430 .187210 .25493$

EDGE $0.480830 .135100 .046290 .495320 .14394-0.45354$

EDGE $0.230420 .009180 .25467 \quad 0.245040 .017600 .75463$

EDGE $0.230420 .009180 .25467 \quad 0.245040 .01760-0.24537$

EDGE 0.253740 .443530 .080690 .262290 .442550 .59762

EDGE $0.297180 .432660 .12040 \quad 0.311660 .441500 .62023$

EDGE $0.252860 .478880 .22062 \quad 0.217850 .483670 .56624$

EDGE $0.266910 .238250 .07830 \quad 0.227440 .245040 .24537$

EDGE $0.230420 .009180 .25467 \quad 0.23825-0.02865-0.07830$

EDGE $0.441500 .129830 .37977 \quad 0.495320 .143940 .54646$

EDGE $0.203540 .474970 .05733 \quad 0.217850 .483670 .56624$

EDGE $0.297180 .432660 .12040 \quad 0.311660 .44150-0.37977$

EDGE $0.227440 .245040 .24537 \quad 0.259570 .302290 .29192$

EDGE 0.343020 .057580 .075520 .400530 .086220 .04667

EDGE $0.037430 .187210 .25493-0.011500 .171750 .42128$

EDGE $0.480830 .135100 .046290 .432660 .13547-0.12040$

EDGE $0.044190 .233510 .08810 \quad 0.028650 .26691-0.07830$

EDGE $0.343020 .057580 .07552 \quad 0.302290 .04272-0.29192$

EDGE $0.266910 .238250 .07830 \quad 0.225080 .195520 .41205$

EDGE 0.480830 .135100 .046290 .441500 .129830 .37977

EDGE $0.297180 .432660 .12040 \quad 0.351080 .446750 .28699$

EDGE $0.441500 .129830 .37977 \quad 0.446750 .095670 .71301$

EDGE $0.183250 .011500 .42128 \quad 0.229560 .051030 .58833$ 
EDGE $0.230420 .009180 .25467 \quad 0.287620 .032890 .21595$

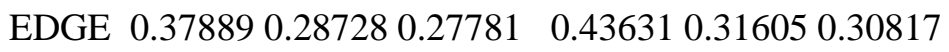

EDGE $0.343020 .057580 .07552 \quad 0.287620 .032890 .21595$

EDGE $0.351080 .446750 .28699 \quad 0.351380 .495320 .45354$

EDGE $0.287620 .032890 .21595 \quad 0.302290 .04272-0.29192$

EDGE $0.230420 .009180 .25467 \quad 0.229560 .051030 .58833$

EDGE $0.137820 .194040 .04498 \quad 0.186370 .191670 .07868$

EDGE $0.227440 .245040 .24537 \quad 0.178530 .229560 .41167$

EDGE $0.480830 .135100 .046290 .446750 .09567-0.28699$

EDGE $0.088380 .189940 .20462 \quad 0.037430 .187210 .25493$

EDGE $0.441130 .081240 .21307 \quad 0.480830 .135100 .04629$

EDGE $0.186370 .191670 .07868 \quad 0.178530 .229560 .41167$

EDGE $0.441130 .081240 .21307 \quad 0.441500 .129830 .37977$

EDGE $0.183250 .011500 .42128 \quad 0.188070 .063580 .44348$

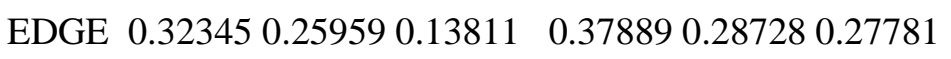

EDGE $0.297180 .432660 .12040 \quad 0.302100 .478250 .28660$

EDGE $0.137820 .194040 .04498 \quad 0.088380 .189940 .20462$

EDGE $0.578740 .102270 .09207 \quad 0.630000 .105630 .14136$

EDGE $0.302100 .478250 .28660 \quad 0.351380 .495320 .45354$

EDGE $0.323450 .259590 .13811 \quad 0.364360 .27646-0.22988$

EDGE $0.114530 .470030 .35833 \quad 0.168590 .484670 .52507$

EDGE $0.088380 .189940 .20462 \quad 0.124490 .188070 .55652$

EDGE $0.480830 .135100 .046290 .478250 .17615-0.28660$

EDGE $0.087900 .364360 .22988 \quad 0.091610 .37889-0.27781$

EDGE $0.441130 .081240 .21307 \quad 0.446750 .09567-0.28699$

EDGE $0.137820 .194040 .04498 \quad 0.124490 .188070 .55652$

EDGE $0.114530 .470030 .35833 \quad 0.105630 .475630 .85864$

EDGE $0.087900 .364360 .22988 \quad 0.114640 .421770 .19167$

EDGE $0.114530 .470030 .35833 \quad 0.105630 .47563-0.14136$

EDGE $0.441130 .081240 .21307 \quad 0.446750 .095670 .71301$

EDGE $0.087340 .222030 .08007 \quad 0.044190 .233510 .08810$

EDGE $0.266910 .238250 .07830 \quad 0.323450 .259590 .13811$

EDGE $0.441130 .081240 .21307 \quad 0.490390 .098360 .37978$

EDGE $0.087340 .222030 .08007 \quad 0.127930 .228020 .42104$ 
EDGE $0.400530 .086220 .04667 \quad 0.432660 .13547-0.12040$

EDGE $0.490390 .098360 .37978 \quad 0.542670 .104430 .44259$

EDGE $0.154090 .475750 .02523 \quad 0.168590 .48467-0.47493$

EDGE $0.127930 .228020 .42104 \quad 0.178530 .229560 .41167$

EDGE $0.228340 .147360 .40685 \quad 0.225080 .195520 .41205$

EDGE $0.578740 .102270 .09207 \quad 0.542670 .104430 .44259$

EDGE $0.490390 .098360 .37978 \quad 0.446750 .095670 .71301$

EDGE $0.154090 .475750 .02523 \quad 0.168590 .484670 .52507$

EDGE 0.107540 .515930 .395520 .104430 .561760 .55741

EDGE $0.087340 .222030 .08007 \quad 0.080980 .228340 .59315$

EDGE $0.490390 .098360 .37978 \quad 0.495320 .143940 .54646$

EDGE $0.578740 .102270 .09207 \quad 0.591610 .107540 .60448$

EDGE $0.114530 .470030 .35833 \quad 0.107540 .515930 .39552$

EDGE $0.154090 .475750 .02523 \quad 0.114530 .470030 .35833$

EDGE 0.480830 .135100 .046290 .490390 .098360 .37978

EDGE $0.154090 .475750 .02523 \quad 0.105630 .47563-0.14136$

EDGE $0.228340 .147360 .40685 \quad 0.228020 .100080 .57896$

EDGE $0.441500 .129830 .37977 \quad 0.478250 .176150 .71340$

\# EDGE_CENTER 0.134370 .448760 .10845

\# EDGE_CENTER 0.423640 .09094 -0.12016

\# EDGE_CENTER 0.114580 .445900 .27500

\# EDGE_CENTER 0.564730 .146120 .39026

\# EDGE_CENTER 0.179060 .187460 .32870

\# EDGE_CENTER 0.539220 .142190 .31099

\# EDGE_CENTER 0.520600 .144660 .55663

\# EDGE_CENTER 0.460490 .316060 .39155

\# EDGE_CENTER 0.17906 0.18746-0.17130

\# EDGE_CENTER 0.137180 .45603 -0.14147

\# EDGE_CENTER 0.558060 .142930 .13444

\# EDGE_CENTER 0.421020 .108030 .21322

\# EDGE_CENTER 0.117390 .453170 .52508

\# EDGE_CENTER 0.455970 .343020 .22477

\# EDGE_CENTER 0.420830 .083730 .12987

\# EDGE_CENTER 0.60908 0.148950.26109 
\# EDGE_CENTER 0.506700 .137050 .05073

\# EDGE_CENTER 0.117450 .429040 .44175

\# EDGE_CENTER $0.036880 .22930-0.16197$

\# EDGE_CENTER 0.117450 .42904 -0.05825

\# EDGE_CENTER 0.397130 .003770 .11372

\# EDGE_CENTER 0.364410 .431480 .25053

\# EDGE_CENTER 0.500560 .340750 .39170

\# EDGE_CENTER 0.036880 .229300 .33803

\# EDGE_CENTER 0.205730 .193600 .24536

\# EDGE_CENTER 0.152570 .49610 -0.14163

\# EDGE_CENTER 0.425270 .052850 .25441

\# EDGE_CENTER 0.18939-0.009030.50461

\# EDGE_CENTER 0.019440 .209940 .00471

\# EDGE_CENTER 0.132780 .493240 .52493

\# EDGE_CENTER 0.045280 .41400 -0.02198

\# EDGE_CENTER 0.496040 .367710 .22492

\# EDGE_CENTER 0.094090 .448130 .19134

\# EDGE_CENTER 0.442020.155040.39107

\# EDGE_CENTER 0.20381 0.21105 -0.08799

\# EDGE_CENTER 0.234340 .442750 .25095

\# EDGE_CENTER 0.096960 .43127 -0.14191

\# EDGE_CENTER 0.226260 .220280 .32871

\# EDGE_CENTER 0.089640 .45093 -0.05850

\# EDGE_CENTER 0.094150 .424000 .10801

\# EDGE_CENTER 0.189480 .440600 .38988

\# EDGE_CENTER 0.275460 .438090 .10055

\# EDGE_CENTER 0.017510 .227380 .17139

\# EDGE_CENTER 0.206840 .010340 .33797

\# EDGE_CENTER 0.166300 .461950 .44181

\# EDGE_CENTER 0.21297 -0.01019 0.42131

\# EDGE_CENTER 0.389750 .400530 .14119

\# EDGE_CENTER 0.412810 .031470 .04084

\# EDGE_CENTER 0.484170 .290950 .45434

\# EDGE_CENTER 0.033490 .206150 .42144 
\# EDGE_CENTER 0.159050.457490 0.19189 \# EDGE_CENTER 0.228200 .476920 .13897 \# EDGE_CENTER $0.186790 .17073-0.08812$ \# EDGE_CENTER 0.139330 .430500 .27510 \# EDGE_CENTER 0.142140 .437770 .52518 \# EDGE_CENTER 0.277480 .478560 .25361 \# EDGE_CENTER 0.488080 .139520 .29637 \# EDGE_CENTER 0.178820 .475360 .04128 \# EDGE_CENTER 0.206900 .218360 .16203 \# EDGE_CENTER 0.442260 .203640 .49058 \# EDGE_CENTER 0.04081 0.210360.17151 \# EDGE_CENTER 0.488080 .13952 -0.20363 \# EDGE_CENTER 0.237730 .013390 .50465 \# EDGE_CENTER 0.237730 .013390 .00465 \# EDGE_CENTER 0.258020 .443040 .33916 \# EDGE_CENTER 0.304420 .437080 .37031 \# EDGE_CENTER 0.235350 .481270 .39343 \# EDGE_CENTER 0.24717 0.241640.16184 \# EDGE_CENTER 0.23434 -0.009740.08818 \# EDGE_CENTER 0.468410 .136890 .46311 \# EDGE_CENTER 0.210700 .479320 .31178 \# EDGE_CENTER 0.304420 .43708 -0.12969 \# EDGE_CENTER 0.24351 0.27367 0.26864 \# EDGE_CENTER 0.371780 .071900 .06110 \# EDGE_CENTER 0.012960 .179480 .33810 \# EDGE_CENTER 0.45674 0.13529-0.03705 \# EDGE_CENTER 0.036420 .250210 .00490 \# EDGE_CENTER 0.322660 .05015 -0.10820 \# EDGE_CENTER 0.24599 0.216890.24517 \# EDGE_CENTER 0.461160 .132470 .21303 \# EDGE_CENTER 0.324130 .439700 .20370 \# EDGE_CENTER 0.444120 .112750 .54639 \# EDGE_CENTER 0.206410.031270.50480 \# EDGE_CENTER 0.259020 .021030 .23531 
\# EDGE_CENTER 0.407600 .301660 .29299 \# EDGE_CENTER 0.315320 .045230 .14573 \# EDGE_CENTER 0.351230 .471030 .37027 \# EDGE_CENTER 0.294960.03780 -0.03798 \# EDGE_CENTER 0.229990 .030100 .42150 \# EDGE_CENTER 0.162100 .192860 .06183 \# EDGE_CENTER 0.202980 .237300 .32852 \# EDGE_CENTER 0.463790 .11538 -0.12035 \# EDGE_CENTER 0.062900 .188580 .22977 \# EDGE_CENTER 0.460980 .108170 .12968 \# EDGE_CENTER 0.182450 .210620 .24518 \# EDGE_CENTER 0.441320 .105540 .29642 \# EDGE_CENTER 0.185660 .037540 .43238 \# EDGE_CENTER 0.351170 .273430 .20796 \# EDGE_CENTER 0.299640 .455450 .20350 \# EDGE_CENTER 0.113100 .191990 .12480 \# EDGE_CENTER 0.604370.103950.11671 \# EDGE_CENTER 0.326740 .486780 .37007 \# EDGE_CENTER 0.343910 .26802 -0.04589 \# EDGE_CENTER 0.141560 .477350 .44170 \# EDGE_CENTER 0.106430 .189010 .38057 \# EDGE_CENTER 0.479540 .15563 -0.12016 \# EDGE_CENTER 0.089750 .37162 -0.02397 \# EDGE_CENTER 0.443940 .08845 -0.03696 \# EDGE_CENTER 0.131160 .191060 .30075 \# EDGE_CENTER 0.110080 .472830 .60849 \# EDGE_CENTER 0.10127 0.393070.21077 \# EDGE_CENTER 0.110080 .472830 .10849 \# EDGE_CENTER 0.443940 .088450 .46304 \# EDGE_CENTER 0.065760 .227770 .08409 \# EDGE_CENTER 0.295180 .248920 .10820 \# EDGE_CENTER 0.465760 .089800 .29643 \# EDGE_CENTER 0.10764 0.22503 0.25055 \# EDGE_CENTER 0.416600 .11085 -0.03686 
\# EDGE_CENTER 0.516530 .101400 .41118 \# EDGE_CENTER 0.161340 .48021 -0.22485 \# EDGE_CENTER 0.15323 0.228790.41636 \# EDGE_CENTER 0.226710 .171440 .40945 \# EDGE_CENTER 0.560700 .103350 .26733 \# EDGE_CENTER 0.468570 .097010 .54640 \# EDGE_CENTER 0.161340 .480210 .27515 \# EDGE_CENTER 0.105990 .538850 .47647 \# EDGE_CENTER 0.08416 0.225190.33661 \# EDGE_CENTER 0.492850 .121150 .46312 \# EDGE_CENTER 0.585170 .104910 .34828 \# EDGE_CENTER 0.111030 .492980 .37692 \# EDGE_CENTER 0.134310.472890.19178 \# EDGE_CENTER 0.485610 .116730 .21304 \# EDGE_CENTER 0.12986 0.47569 -0.05806 \# EDGE_CENTER 0.228180 .123720 .49290 \# EDGE_CENTER 0.459870 .152990 .54659 END 